\title{
Cisplatin enhances apoptosis induced by a tumor-selective adenovirus expressing tumor necrosis factor-related apoptosis-inducing ligand
}

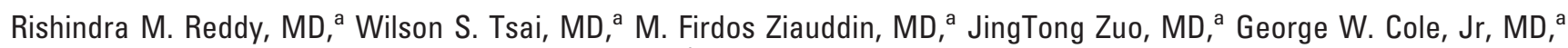
Justin B. Maxhimer, BA, ${ }^{\text {a }}$ BingLiang Fang, MD, PhD, ${ }^{b}$ David S. Schrump, MD, FACS ${ }^{a}$, and

Dao M. Nguyen, MD, FRCSC, FACS ${ }^{a}$

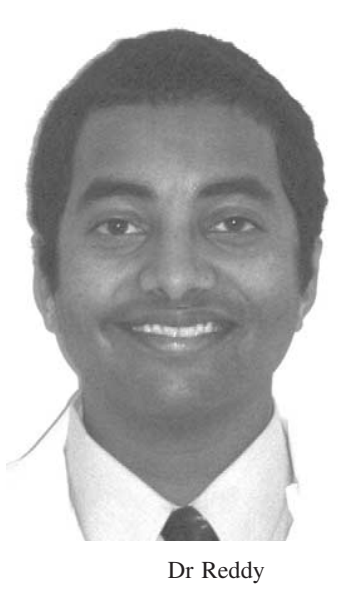

From the Section of Thoracic Oncology, ${ }^{\mathrm{a}}$ Surgery Branch, Center for Cancer Research, National Cancer Institute, National Institutes of Health, Bethesda, Md, and the Department of Thoracic and Cardiovascular Surgery, , University of Texas MD Anderson Cancer Center, Houston, Tex.

Read at the Eighty-fourth Annual Meeting of the American Association for Thoracic Surgery, Toronto, Ontario, Canada, April 25-28, 2004.

Received for publication April 22, 2004; revisions received June 7, 2004; accepted for publication June 16, 2004

Address for reprints: Dao M. Nguyen, MD, NCI/NIH, Room 2B07, Building 10, 10 Center Dr, Bethesda, MD 20892 (E-mail: Dao_Nguyen@nih.gov).

J Thorac Cardiovasc Surg 2004;128:883-91 $0022-5223 / \$ 30.00$

Copyright (c) 2004 by The American Association for Thoracic Surgery

doi:10.1016/j.jtcvs.2004.06.036
Background: Cancer cells frequently exhibit resistance to the cytotoxic effect of tumor necrosis factor-related apoptosis-inducing ligand (TRAIL). Pretreatment of TRAIL-resistant cells with cisplatin sensitizes them to this ligand. Cisplatin also has been shown to enhance adenoviral transgene expression.

Objective: This study aims to evaluate the ability of cisplatin to enhance the expression and the cytotoxic effect of the tumor-specific adenoviral vector $\mathrm{Ad} /$ gTRAIL, which expresses a green fluorescent protein-TRAIL fusion protein.

Methods: Cultured cancer cells and normal human cells were infected with Ad/ gTRAIL with or without cisplatin pretreatment. Adenoviral transgene expression was determined by using flow cytometry to measure green fluorescent protein fluorescence. Cytotoxicity was measured by using thiazolyl blue tetrazolium bromide assays and an apoptosis enzyme-linked immunosorbent assay kit.

Results: Green fluorescent protein-TRAIL fusion protein expression was significantly enhanced by cisplatin pretreatment in cancer cells. Cisplatin treatment before Ad/gTRAIL infection resulted in a 2- to 12-fold increase in green fluorescent protein fluorescence intensity across cancer lines. Although Ad/gTRAIL induced mild cytotoxicity in all cancer lines (inhibitory concentration of 50\% values of $>500$ pfu/cell), pretreatment with cisplatin resulted in a dose-dependent enhancement of Ad/gTRAIL-mediated cytotoxicity, as indicated by the drastic reduction of inhibitory concentration of 50\% values to 4 to $42 \mathrm{pfu} / \mathrm{cell}$ in all cell lines. There was no cytotoxicity noted in normal cells treated with both cisplatin and Ad/gTRAIL.

Conclusion: Cisplatin pretreatment enhances Ad/gTRAIL cytotoxicity in malignant cells while not affecting normal cells. The mechanisms underlying this effect might include both enhancement of the susceptibility of cisplatin-treated cells to TRAIL and cisplatin-mediated enhancement of TRAIL expression in Ad/gTRAIL infected cells. These findings provide a rationale for development of Ad/gTRAIL-based therapy for thoracic malignancies.

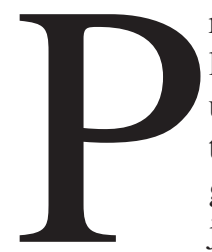
rimary cancers of the thoracic cavity, those that originate from the lung, the esophagus, and the pleura, remain the most common and, unfortunately, the most resistant to standard multimodality combinations of surgical intervention, chemotherapy, and radiotherapy. Aggressive systemic therapy with cytotoxic agents might provide subjective responses, yet patients are plagued with severe side effects. The mechanism of resistance to chemotherapy or radiotherapy in malignant cells is multifactorial and incompletely understood. Better appreciation of the molecular basis of tumorigenesis, particularly signal transduction pathways that govern cell 
growth and death, provide unique opportunities for the development of molecular therapeutic approaches that selectively target cancer cells and not normal cells.

Receptor-mediated apoptosis-inducing pathways controlled by members of the tumor necrosis factor (TNF) superfamily, particularly those controlled by TNF-related apoptosis-inducing ligand (TRAIL), attract significant attention and are currently undergoing preclinical development. ${ }^{1}$ Binding of TRAIL to its cognate receptors, DR4 or DR5, initiates a well-defined cascade of caspase activation, leading to a rapid and profound induction of apoptosis in susceptible malignant cells while sparing normal cells. ${ }^{2}$ The gene encoding human TRAIL has been cloned, and the soluble recombinant protein has been shown to mediate potent antitumor activity both in vitro ${ }^{3}$ and in in vivo preclinical animal experiments. ${ }^{4}$ It is well documented that cytotoxic chemotherapeutic agents synergistically interact with TRAIL to induce apoptosis, even in TRAIL-resistant cancer cells. ${ }^{5,6}$ We have shown the DNA-damaging agent cisplatin to be a potent chemoenhancer of soluble Fas ligand-mediated apoptosis ${ }^{7}$ and Apo2L/TRAIL-mediated apoptosis. ${ }^{8}$ in various thoracic malignancies. Even with these promising results, there has been concern regarding hepatotoxicity with systemic administration. ${ }^{9}$

Adenovirus is a commonly used vector to deliver therapeutic genes to eukaryotes. It is limited by its tropism for hepatocytes and its indiscriminate infectivity in both cancer cells and normal cells. ${ }^{10}$ Adenovirus expressing the membrane-bound green fluorescent protein (GFP)-TRAIL fusion protein under the control of the human telomerase reverse transcriptase (hTERT) promoter (Ad/gTRAIL) has been shown to be selectively active in transformed, but not normal, cells because hTERT is overexpressed in up to $90 \%$ of cancer cells. This vector has also been evaluated in normal human hepatocytes and shown to initiate minimal TRAIL expression, and it has no cytotoxicity. ${ }^{11}$ These features make such a recombinant adenovirus an attractive vector for cancer gene therapy. Moreover, it has been demonstrated that pretreating cancer cells with subtherapeutic doses of cisplatin would result in significant enhancement of adenovirally mediated transgene expression and function of therapeutic genes. ${ }^{12}$

In this study we evaluated the enhanced transgene expression and the cytotoxic effect of Ad/gTRAIL after cisplatin treatment in a panel of cultured cancer cells derived from cancers of the lung (non-small cell lung cancer [NSCLC]), the esophagus (esophageal cancer [EsC]), and the pleura (malignant pleural mesothelioma $[\mathrm{MPM}]$ ). We also tested the safety of these agents in normal human cells. Combining Ad/gTRAIL with cisplatin would exploit 2 potentially beneficial effects of cisplatin, the enhancement of adenoviral transgene expression in effector cells and the potentiation of a TRAIL effect on target cells, and this might result in a synergistic therapeutic effect.

\section{Materials and Methods \\ Cells and Reagents}

Cultured NSCLC cells (H322), EsC cells (TE2 and TE12), and MPM cells (H513, H2373, and H28) were maintained in RPMI1640 medium supplemented with glutamine $(1 \mathrm{mmol} / \mathrm{L})$, streptomycin $(100 \mathrm{mg} / \mathrm{mL})$, penicillin $(100 \mathrm{U} / \mathrm{mL})$, and fetal calf serum (5\% vol/vol; complete media). Normal human fibroblasts, human umbilical vein endothelial cells, and normal human keratinocytes were purchased from Clonetics Corp (Walkerville, Md) and cultured with appropriate media as per the instructions of the vendor. Cisplatin (in aqueous solution at $1 \mathrm{mg} / \mathrm{mL}$ ) was purchased from American Pharmaceutical Products (Schaumburg, Ill) and handled in subdued light conditions. Adenovirus vectors expressing GFPTRAIL-Ad/gTRAIL (titer, $5.3 \times 10^{9} \mathrm{pfu} / \mathrm{mL}$ ) and GFP vector control-Ad/GFP (titer, $3.5 \times 10^{9} \mathrm{pfu} / \mathrm{mL}$ ) were obtained from the W. M. Keck Center for Cancer Gene Therapy-Vector Core Laboratory at MD Anderson Cancer Center (Houston, Tex). Biotinylated anti-DR4, anti-DR5, IgG isotype control antibodies, and strepavidin-phycoerythrin were purchased from R\&D Systems (Minneapolis, Minn) and Becton-Dickinson (Torrance, Calif), respectively, and used for receptor expression analysis. The general caspase inhibitor Z-VAD-fmk was purchased from R\&D Systems. DAPI (4',6-diamidino-2-phenylindole), used for nuclear staining, came from Calbiochem (San Diego, Calif), and thiazolyl blue tetrazolium bromide came from Sigma-Aldrich (St Louis, Mo).

\section{Receptor Expression Analysis}

Cells, seeded $10^{6} /$ well in a $60-\mathrm{mm}$ dish and after overnight incubation, were treated with cisplatin $(2 \mu \mathrm{g} / \mathrm{mL})$ or complete media for 24 hours. Cells were then trypsinized, washed with media, and incubated with anti-DR4, anti-DR5, or IgG isotype antibodies $\left(1 \mu \mathrm{g}\right.$ for $3 \times 10^{5}$ cells) for 1 hour. Cells were washed and incubated with strepavidin-phycoerythrin for 30 minutes in the dark and submitted for flow cytometric analysis.

\section{Flow Cytometric Analysis of GFP and GFP-TRAIL Expression}

Cells, seeded at $10^{5}$ /well in 12-well plates and after an overnight incubation, were either treated with cisplatin $(1-2 \mu \mathrm{g} / \mathrm{mL})$ or complete media (control cells) for 24 hours. Control or cisplatintreated cells were infected with 6 or 12 pfu/cell of either Ad/GFP or Ad/gTRAIL, respectively. The caspase inhibitor Z-VAD-fmk ( $60 \mu \mathrm{mol} / \mathrm{L}$ ) was used to prevent caspase-mediated apoptosis of Ad/gTRAIL-infected cells, particularly of those pretreated with cisplatin. Cells were trypsinized, washed with fresh media 48 hours after adenovirus infection, and submitted for analysis of GFP expression (which correlates with TRAIL expression for $\mathrm{Ad} /$ gTRAIL) by means of flow cytometry. Imaging of cells for GFP expression was done 24 to 48 hours after Ad/gTRAIL or Ad/GFP infection by using fluorescence microscopy.

\section{Ad/gTRAIL-mediated Cytotoxicity}

Cells were seeded at $10^{4}$ cells/well in flat-bottom 96-well plates. After an overnight incubation, they were either incubated with normal media or with cisplatin $(0.5,1$, and $2 \mu \mathrm{g} / \mathrm{mL})$ for 24 hours. Cells were subsequently infected with Ad/gTRAIL (0, 3, 6, 12, 25, and $50 \mathrm{pfu} / \mathrm{cell})$ or Ad/GFP (50 pfu/cell). Cell viability at 60 hours after adenovirus treatment was quantitated by using the thiazolyl 

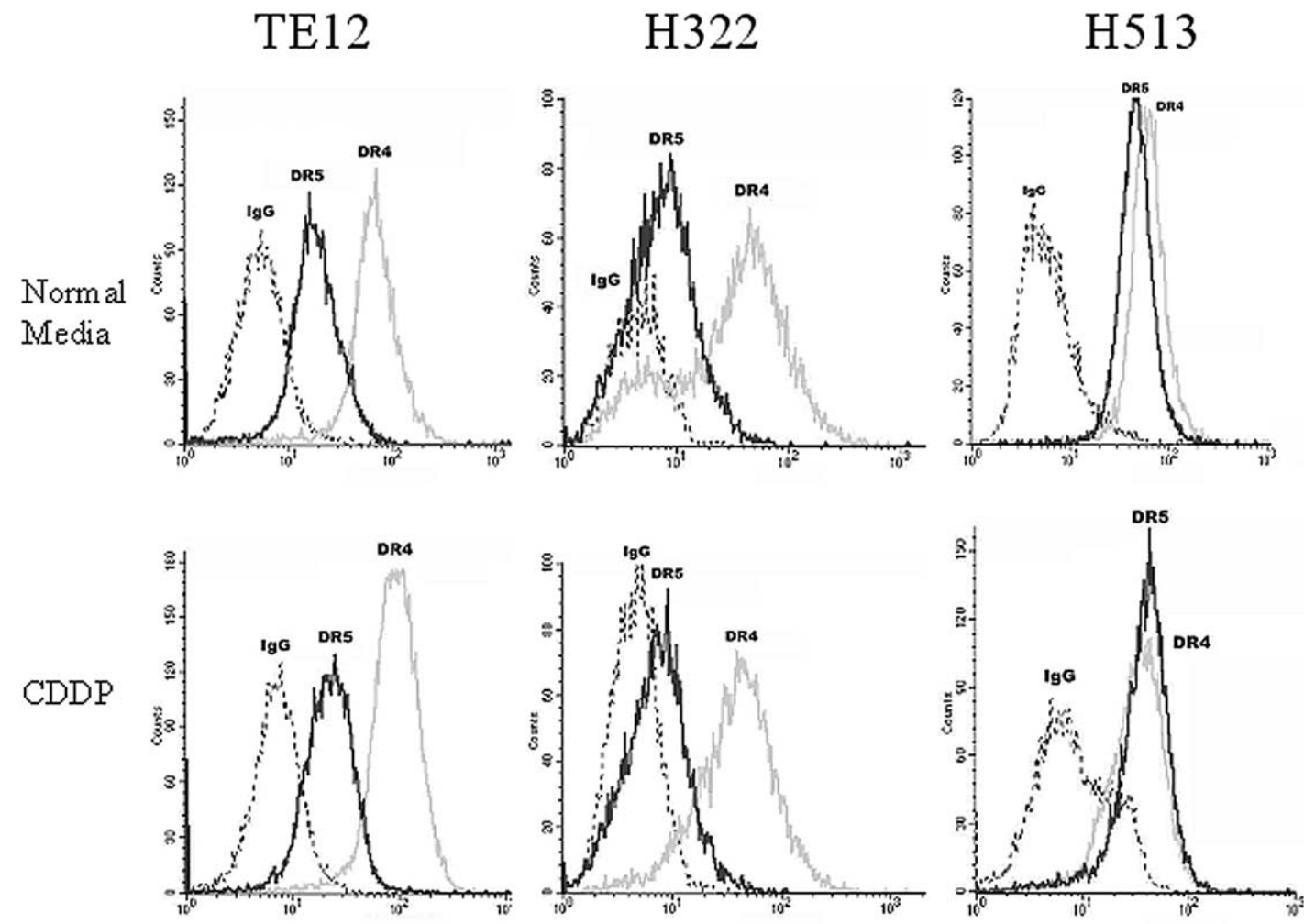

Figure 1. Flow cytometric analysis of DR4 and DR5 expression on the cultured cancer cells TE12, H322, and H513. Exposure of these cells to cisplatin for 24 hours did not affect the levels of DR4 or DR5 expression. Representative data from 3 independent experiments with similar results are shown.

blue tetrazolium bromide assay. Cell viability after Ad/gTRAIL or cisplatin/Ad/gTRAIL treatments was expressed as a percentage of cell viability of the appropriate controls (those with or without cisplatin exposure that were not infected with Ad/gTRAIL to normalize for the mild growth-inhibitory effect of cisplatin pretreatment).

\section{Evaluation of Apoptosis}

Cells were plated at $10^{5}$ cells/well in 12 -well plates. After an overnight incubation, they were treated with complete media or cisplatin $(1 \mu \mathrm{g} / \mathrm{mL})$ for 24 hours and then infected with $\mathrm{Ad} /$ gTRAIL (12-25 pfu/cell). Cells were harvested 24 or 48 hours after adenovirus infection and assayed for apoptosis-induced DNA fragmentation resulting in measurable oligonucleosome cytoplasmic release by using the Cell Death Detection ELISA kit (Roche, Indianapolis, Ind). The standard fluorescein isothiocyanatedependent apoptosis assay techniques (TUNEL or Annexin V) could not be used in this study because Ad/gTRAIL-infected cells express GFP, which interferes with fluorescein isothiocyanate detection by means of flow cytometry. Apoptosis-induced DNA fragmentation was also visualized in cells treated with cisplatin plus Ad/GFP or Ad/gTRAIL combinations by means of DAPI staining and fluorescence microscopy.

\section{Results}

\section{DR4/DR5 Expression in Thoracic Malignancies}

All cultured cancer cell lines evaluated abundantly expressed DR4, DR5, or both, as determined by means of indirect immunofluorescent staining and flow cytometry. Cisplatin $(2 \mu \mathrm{g} / \mathrm{mL} \times 24$ hours $)$ treatment did not alter the baseline DR4 or DR5 expression of any of the cultured cancer cells. Representative data are shown for H513 (MPM), H322 (NSCLC), and TE12 (EsC) cells (Figure 1). Similarly, primary normal cells also expressed these 2 TRAIL receptors, and their levels also did not change with cisplatin exposure (data not shown).

\section{Cisplatin-mediated Enhancement of GFP-TRAIL Expression}

Baseline expression of GFP-TRAIL fusion protein after Ad/gTRAIL infection was dependent on the individual cell line. Significant GFP-TRAIL expression was noted in H513 and $\mathrm{H} 28$ cells, whereas it was barely detectable in other cell lines (Figure 2, A). Titration experiments performed for all cancer cell lines indicated a cell line-dependent correlation 


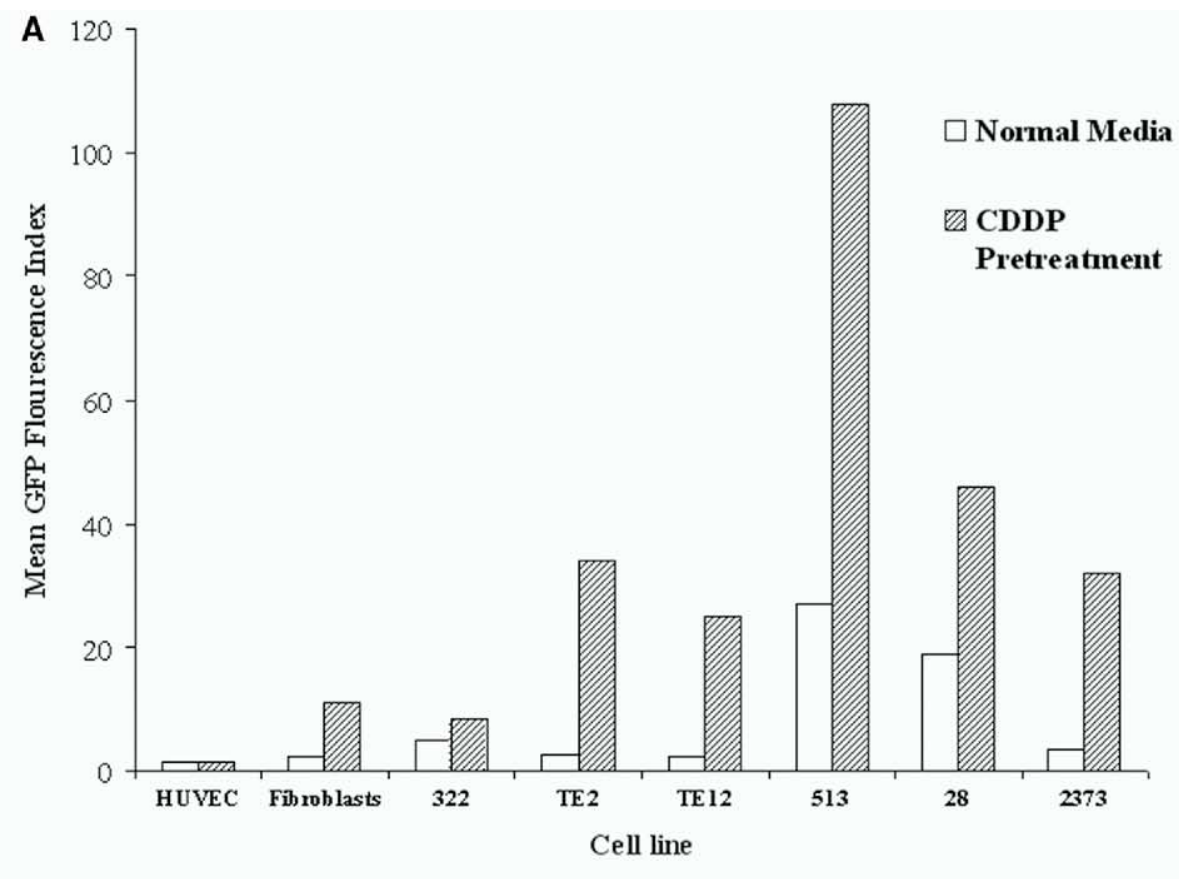

B $\quad$ CDDP
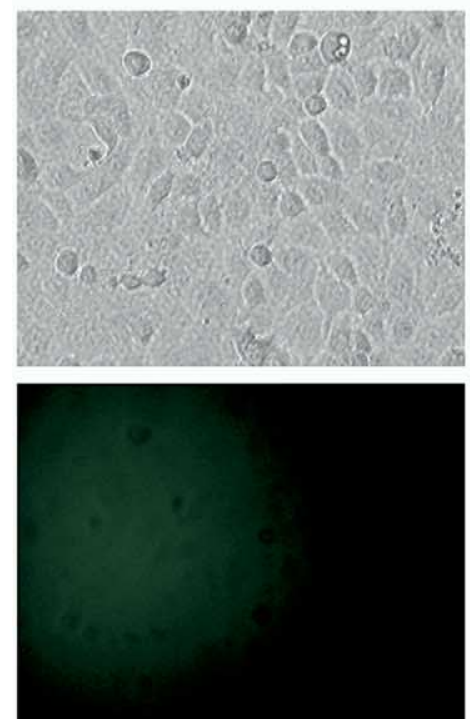

Ad/gTRAIL

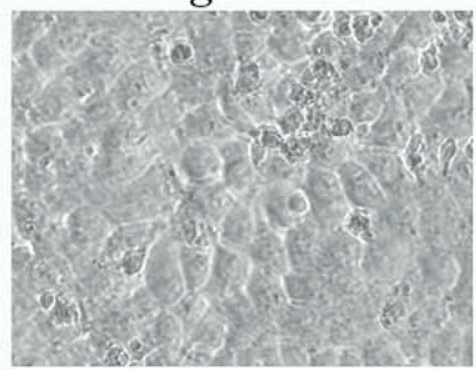

CDDP + Ad/gTRAIL
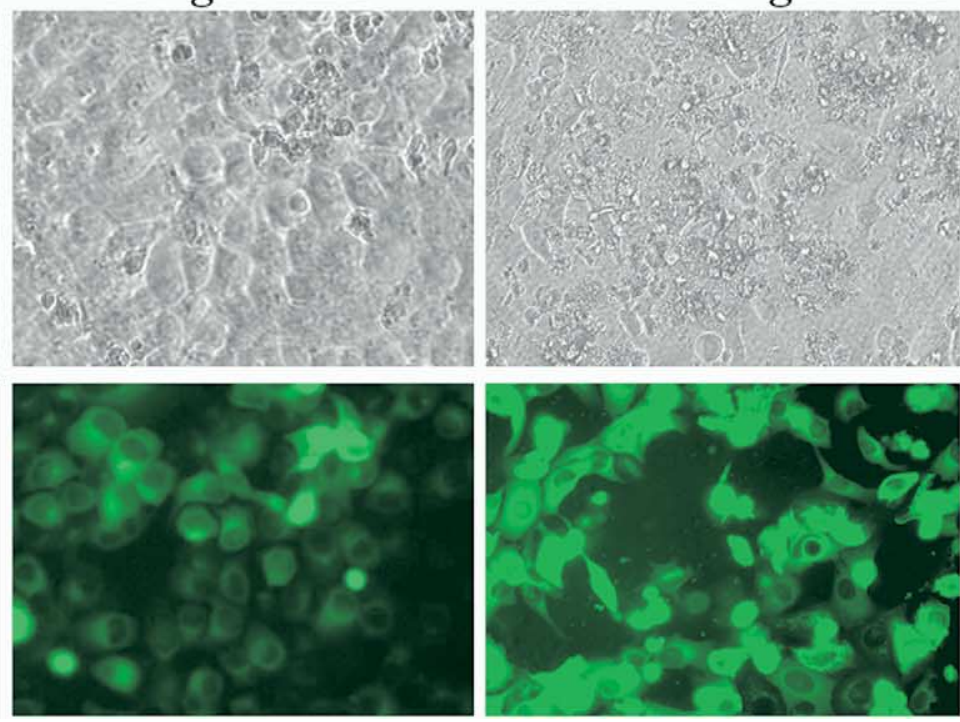

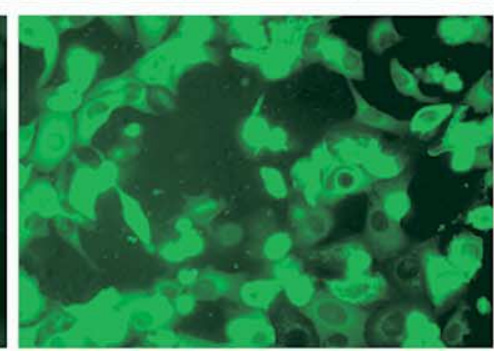

Figure 2. A, Expression of GFP-TRAIL after Ad/gTRAIL infection (12 pfu/cell), as measured on the basis of the green fluorescence intensity index (GFP mean fluorescence intensity of Ad/gTRAIL-infected cells normalized for background fluorescence) in cultured cancer cells and primary normal cells. Significantly enhanced adenovirusmediated GFP-TRAIL expression in cultured cancer cells and, to a much lesser degree, in primary normal cells was seen by treating cells with cisplatin $(1 \mu \mathrm{g} / \mathrm{mL})$ for 24 hours before Ad/gTRAIL infection. B, Fluorescence microscopy with a GFP filter setting of $\mathrm{H} 322$ cells treated with control media, Ad/gTRAIL (25 pfu), and cisplatin (1 $\mu \mathrm{g} / \mathrm{mL}$ ) plus Ad/gTRAIL.

between increasing GFP expression and increasing adenoviral dose (data not shown). Treating cultured cancer cells with a nontoxic concentration of cisplatin $(1 \mu \mathrm{g} / \mathrm{mL})$ for 24 hours before Ad/gTRAIL infection significantly enhanced the GFP-TRAIL transgene expression, with the increase of the GFP fluorescence intensity ranging from 2-fold (H322) to 12-fold (TE2). It appeared that cell lines with low baseline expression of GFP-TRAIL (TE2, TE12, and H2373) had the highest degrees of gene upregulation versus cell lines that had higher baseline expression of the transgene (as 

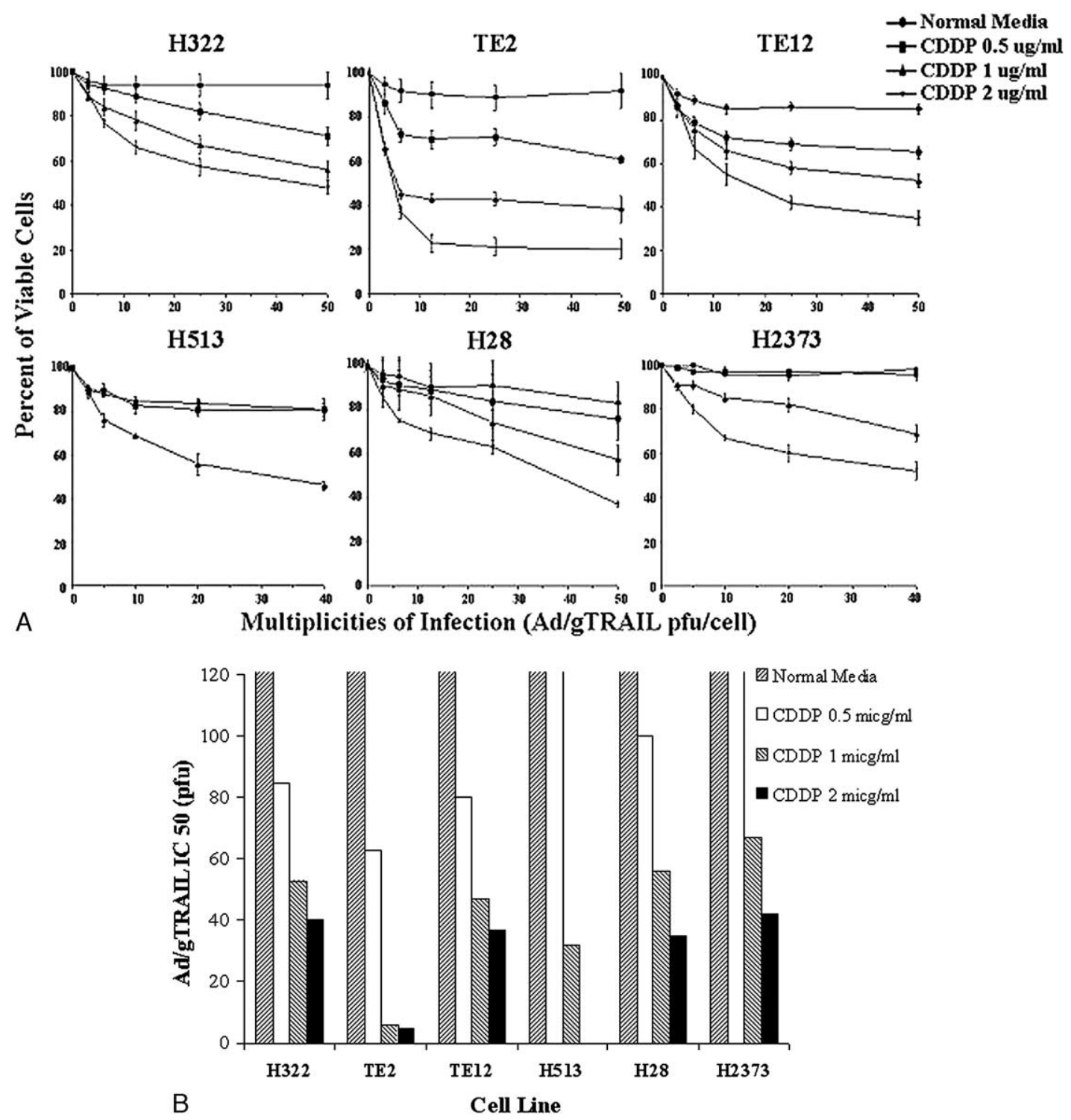

Figure 3. A, Cisplatin-mediated enhancement of Ad/gTRAIL cytotoxicity in cultured cancer cells. Percentages of viable cells were calculated after normalization for the mild growth-inhibitory effect of cisplatin exposure. Data are expressed as means \pm SEM of 4 independent experiments. By using an unpaired $t$ test evaluating for 2-tailed $\boldsymbol{P}$ values to compare the highest doses of Ad/gTRAlL with or without cisplatin, we had $\boldsymbol{P}$ values of .0167 for $\mathrm{H28}$, and $\boldsymbol{P}$ values of less than .001 for the remaining cell lines. B, Ad/gTRAIL IC50 values (the multiplicity of infection of Ad/gTRAIL that mediated a $50 \%$ reduction of cell viability) were derived from the respective dose-response curves.

in H513 and H28). Normal fibroblasts and human umbilical vein endothelial cells showed a very low level of GFPTRAIL expression after Ad/gTRAIL infection, as expected because of the tumor-selective nature of this vector. Pretreating normal cells with cisplatin caused slight enhancement of their GFP fluorescence intensity. Cisplatin-mediated upregulation of GFP-TRAIL expression was readily visualized by mean of fluorescence microscopy (Figure 2,B).
Cisplatin-mediated Enhancement of Ad/gTRAIL Cytotoxicity and Apoptosis

Ad/gTRAIL infection of up to $50 \mathrm{pfu} / \mathrm{cell}$ exerted little cytotoxicity in all cell lines. Treating cultured cancer cells with subtherapeutic doses of cisplatin $(0.5-2 \mu \mathrm{g} / \mathrm{mL})$ for 24 hours profoundly enhanced the Ad/gTRAIL cytotoxic effect (Figure 3, A). The Ad/gTRAIL IC50 values (the multiplicity of infection [in plaque-forming units per cell] that causes 


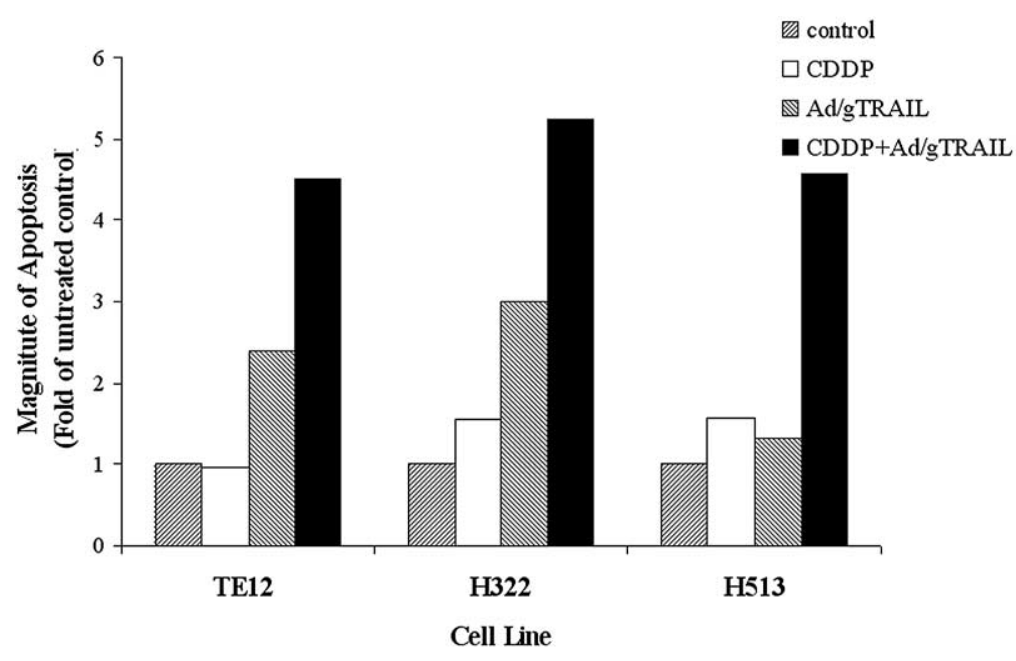

Figure 4. Enhancement of apoptosis of cultured cancer cells treated with sequential cisplatin plus Ad/gTRAIL combinations measured by means of cell-death ELISA. Representative data of 3 independent experiments with similar results are shown.

$50 \%$ inhibition of cell viability) were estimated from respective dose-response curves of cells treated with cisplatin plus Ad/gTRAIL. They showed significant decreases in a cisplatin dose-dependent manner (Figure 3, B). This reduction in IC50 values of Ad/gTRAIL is an indicator of enhanced cellular sensitivity to this TRAIL-expressing virus and resulted in an IC50 value range of 4 pfu/cell (TE2) to 42 pfu/cell (H2373) compared with the IC50 values of cells treated with Ad/gTRAIL alone ( $>500 \mathrm{pfu} / \mathrm{cell}$ in all cell lines). We treated the same panel of cancer cells with the vector control virus $\mathrm{Ad} / \mathrm{GFP}$ at comparable multiplicities of infection with or without cisplatin pretreatment and did not see any cytotoxic effects (data not shown).

Apoptosis of cells treated with cisplatin, Ad/gTRAIL, or the combination of cisplatin followed by Ad/gTRAIL was quantitated by using the DNA fragmentation assay (Figure $4, A)$. The magnitude of increase in DNA fragmentation after treatment was indicated by the fold increase in oligonucleosome cytoplasmic release over the levels of untreated controls. Although cisplatin alone or Ad/gTRAIL alone mediated less than a 3-fold increase of DNA fragmentation, the cisplatin plus Ad/gTRAIL combination induced 4.5-fold to 5-fold increases in apoptosis. Moreover, propidium iodide (PI) staining was also used to evaluate cell viability after treatment. TE2 cells treated with either cisplatin (1 $\mu \mathrm{g} / \mathrm{mL}$ ) or Ad/gTRAIL (12 pfu/cell) were $9 \%$ and $17 \%$ PI positive, respectively, and treating them with the cisplatin plus Ad/gTRAIL combination caused massive cell death, with almost $60 \%$ of the cells being PI positive (data not shown). Cells were also stained with DAPI dye to visualize nuclei undergoing apoptosis after combination treatment. We were able to confirm the lack of toxicity seen with cisplatin alone or cisplatin plus Ad/GFP and visualize the significant apoptosis seen with cisplatin plus Ad/gTRAIL in representative histologies (data not shown).

\section{Tumor Selectivity of the Ad/gTRAIL}

Primary human normal cells (dermal fibroblasts are shown) were similarly infected by the adenoviral GFP control vector, showing strong green fluorescence (Figure 5, A). On the other hand, robust expression of GFP-TRAIL after Adv/ gTRAIL infection was only observed in cancer cells and not in normal cells, indicating that the differential expression of the adenoviral transgene is mediated by the tumor-selective promoter system and not influenced by cellular susceptibility to virus infection. More importantly, all primary normal cells were totally refractory to the cytotoxic effect of $\mathrm{Ad} /$ gTRAIL and the cisplatin plus Ad/gTRAIL combination (Figure 5, B).

\section{Discussion}

The development of novel molecularly targeted therapies for cancer on the basis of the current understanding and new therapeutic paradigms for this deadly disease are actively being pursued to supplement or even potentially replace current ineffective therapies. Activation of receptor-mediated apoptosis pathways with their natural ligands in the form of recombinant proteins is an attractive therapeutic strategy for receptorpositive cancer cells. TRAIL has received a great deal of interest because, in contrast to another member of the TNF superfamily, Fas ligand, it induces apoptosis in cancer cells but not in normal cells. Recombinant human TRAIL, produced as a zinc-containing homotrimer, ${ }^{13}$ has been shown to be safe in a preclinical study of nonhuman primates ${ }^{14}$ and is slated to be used as a systemic therapeutic agent. Investigators are still 
A
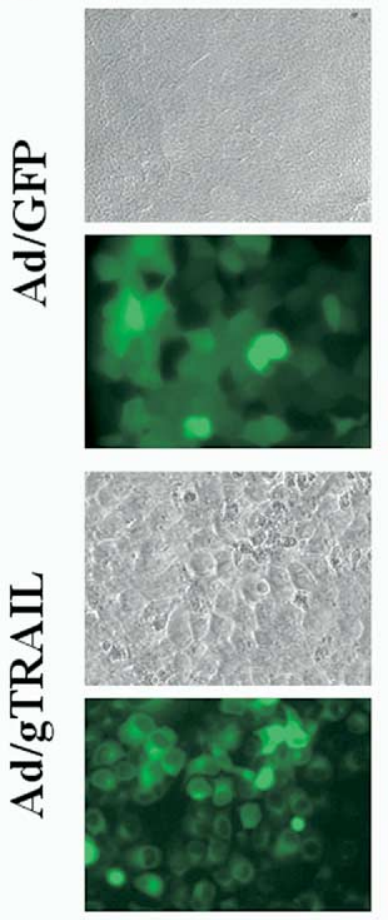

Fibroblasts
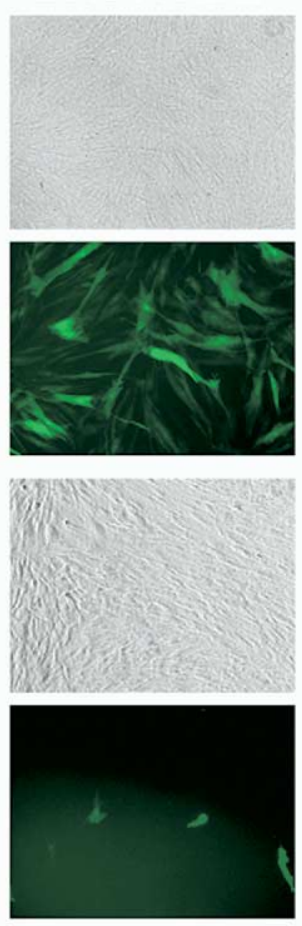

B Fibroblasts
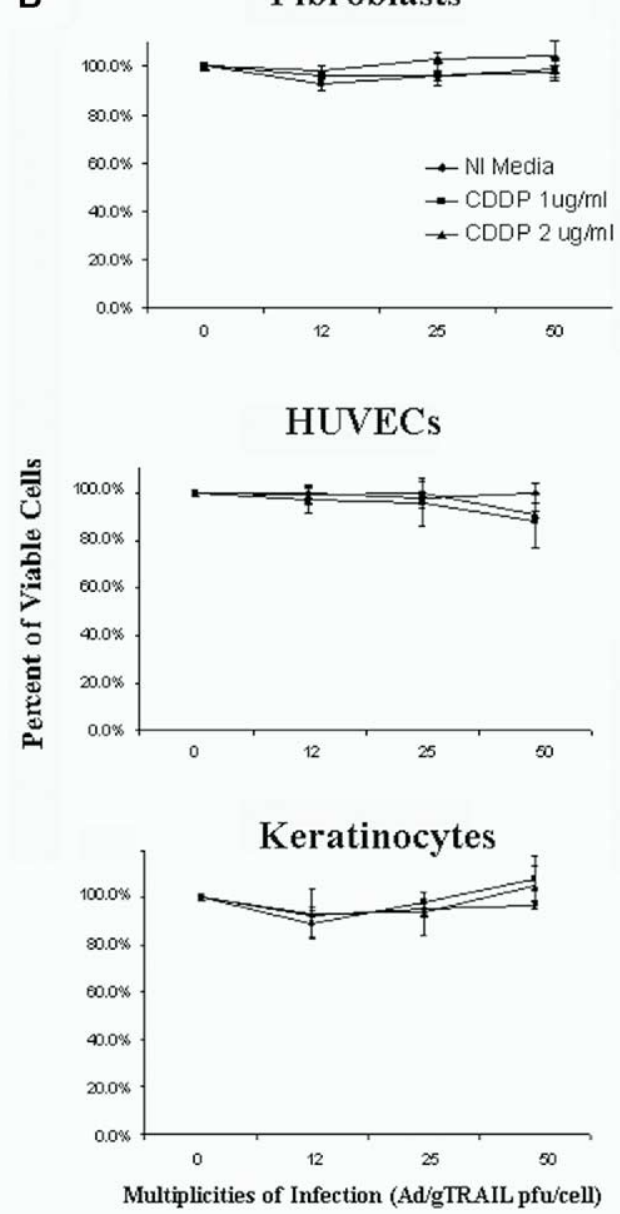

Figure 5. A, Fluorescent microscopy of cultured cancer cells (H322) and human fibroblasts infected with Ad/GFP or Ad/gTRAIL. Normal cells were as efficient as cancer cells in expressing GFP after Ad/GFP infection (25 pfu/cell) but had decreased GFP-TRAIL expression. B, Absence of cytotoxicity in primary normal cells by means of Ad/gTRAIL with or without cisplatin pretreatment. Data are expressed as means \pm SD of 3 independent experiments. HUVEC, Human umbilical vein endothelial cell.

concerned about its potential hepatotoxicity in human subjects. $^{9}$

On the other hand, the gene encoding TRAIL can be directly delivered to tumors by an adenovirus vector to provide high local concentrations of this ligand. Although an excellent vector for efficient gene delivery, adenovirus infects both normal and malignant cells, thus posing a real threat of vector toxicity. Such problems can be minimized by using the hTERT promoter, which is only active in transformed cells and not in normal cells. ${ }^{15}$ In our study we showed the difference between promoter systems because our GFP vector adenovirus uses the constitutively active CMV promoter. This is nonspecific because it is expressed in all cell lines, resulting in similar GFP expression in both cancer cells and normal cells. Fortunately, the Ad/gTRAIL's hTERT-based promoter limits this panexpression. However, a possible drawback is that it does depend on the level of hTERT activity in individual cancer cell lines. The potentially weak transcriptional activity of this tumorspecific promoter associated with the minimal expression of the functional gene might adversely affect the therapeutic utility of such a delivery system. For this reason, we sought to enhance the GFP-TRAIL expression.

Cisplatin pretreatment did not change the tumor selectivity of Ad/gTRAIL. In fact, as seen in a previously reported study, ${ }^{12}$ pretreating cancer cells with subtherapeutic doses of cisplatin before adenoviral infection results in a significant enhancement of transgene expression. The underlying molecular mechanism of this phenomenon has not been clearly elucidated. Increased expression of the coxsackievirus and adenovirus receptor by the histone deacetylase inhibitor depsipeptide has been reported. ${ }^{16}$ It is not known whether cisplatin treatment would mediate similar 
receptor upregulation; further experiments are being conducted to address this possibility. It is conceivable that cisplatin-mediated sublethal DNA damage might activate DNA repair and replication machinery that contributes to the enhanced expression of the adenovirally delivered genes. The magnitude of enhancement of GFP-TRAIL expression was greater in the cells with lower baseline gene expression. This increase in GFP-TRAIL expression in cisplatin-treated cells was likely partially responsible for the profound induction of cytotoxicity and apoptosis in cancer cells that spared normal cells.

The enhanced expression of GFP-TRAIL is not the only benefit of cisplatin pretreatment. Cisplatin has been shown to sensitize TRAIL-resistant cells to the soluble form of this death-inducing ligand. ${ }^{8,17}$ We have found this sensitization effect to have at least a 48-hour window after removal of cisplatin (data not shown). We believe that this sensitization to TRAIL's effects is another method, as important as the increase in adenoviral transgene expression, by which cisplatin enhances the cytotoxic effect of the Ad/gTRAIL on cancer cells. In other words, cisplatin pretreatment, in addition to its ability to increase TRAIL expression on effector cells, also might enhance the responsiveness of target cells whose DR4-DR5 receptors engage membrane-bound TRAIL on the neighboring cells in a paracrine bystander fashion. The relative contribution of each mechanism to the synergistic cytotoxic interaction of the cisplatin plus $\mathrm{Ad} /$ gTRAIL combination is not clear and is certainly difficult to dissect. It is likely cell line dependent as to which mechanism plays the most important role in enhancement.

It is conceivable, however, that adenovirus gene therapy is most suitable for local or regional application. Cisplatinmediated enhancement of the cytotoxic efficacy of $\mathrm{Ad} /$ gTRAIL on cancer cells, while sparing normal cells, tremendously widens the therapeutic index of such a treatment strategy. Functionally speaking, cisplatin can be viewed as a sensitizer of TRAIL and vice versa. It is reasonable to envision use of Ad/gTRAIL for local or regional delivery to bulky cancers in conjunction with systemic cisplatin-based chemotherapy for optimal and synergistic antitumor effects. In vivo experiments with nude mice bearing human xenogafts are being performed to further evaluate this potent combination.

\section{References}

1. Ozoren N, El-Deiry WS. Cell surface death receptor signaling in normal and cancer cells. Semin Cancer Biol. 2003;13:135-47.

2. LeBlanc HN, Ashkenazi A. Apo2L/TRAIL and its death and decoy receptors. Cell Death Differ. 2003;10:66-75.

3. Ashkenazi A, Pai RC, Fong S, Leung S, Lawrence DA, Marsters SA, et al. Safety and antitumor activity of recombinant soluble Apo2 ligand. J Clin Invest. 1999;104:155-62.

4. Kelley SK, Harris LA, Xie D, Deforge L, Totpal K, Bussiere J, et al. Preclinical studies to predict the disposition of Apo2L/tumor necrosis factor-related apoptosis-inducting ligand in humans: characterization of in vivo efficacy, pharmacokinetics, and safety. $J$ Pharmacol Exp Ther. 2001;299:31-8.

5. Lacour S, Hammann A, Wotawa A, Corcos L, Solary E, DimancheBoitrel MT. Anticancer agents sensitize tumor cells to tumor necrosis factor-related apoptosis-inducing ligand-mediated caspase-8 activation and apoptosis. Cancer Res. 2001;61:1645-51.

6. Liu W, Bodle E, Chen JY, Gao M, Rosen GD, Broaddus VC. Tumor necrosis factor-related apoptosis-inducing ligand and chemotherapy cooperate to induce apoptosis in mesothelioma cell lines. Am J Respir Cell Mol Biol. 2001;25:111-8.

7. Stewart JH, Nguyen DM, Chen GA, Schrump DS. Induction of apoptosis in malignant pleural mesothelioma cells by activation of the Fas(Apo-1/CD95) death-signal pathway. J Thorac Cardiovasc Surg. 2002;123:295-302.

8. Tsai WS, Chen GA, Schrump DS, Nguyen DM. Cisplatin potentiates Apo2L/TRAIL-mediated induction of apoptosis in esophageal cancer cells by recruitment of the mitochondria-dependent death signaling pathways [abstract]. Proc Am Assoc Cancer Res. 2003;44:2166.

9. Held J, Schulze-Osthoff K. Potential and caveats of TRAIL in cancer therapy. Drug Resist Updat. 2001;4:243-52.

10. St George JA. Gene therapy progress and prospects: adenoviral vectors. Gene Ther. 2003;10:1135-41.

11. Ling T, Gu J, Zhang L, Huang X, Stephens LC, Curley SA, et al. Targeted expression of green fluorescent protein/tumor necrosis factorrelated apoptosis-inducing ligand fusion protein from human telomerase reverse transcriptase promoter elicits antitumor activity without toxic effects on primary human hepatocytes. Cancer Res. 2002;62: 3620-5.

12. Nguyen DM, Spitz FR, Yen N, Cristiano RJ, Roth JA. Gene therapy for lung cancer: enhancement of tumor suppression by a combination of sequential systemic cisplatin and adenovirus-mediated p53 gene transfer. J Thorac Cardiovasc Surg. 1996;112:1372-6.

13. Hymowitz SG, O'Connell MP, Ultsch MH, Hurst A, Totpal K, Ashkenazi $\mathrm{A}$, et al. A unique zinc-binding site revealed by a high-resolution X-ray structure of homotrimeric Apo2L/TRAIL. Biochemistry. 2000;39:633-40.

14. Lawrence D, Shahrokh Z, Marsters S, Achilles K, Shih D, Mounho B, et al. Differential hepatocyte toxicity of recombinant Apo2L/TRAIL versions. Nat Med. 2001;7:383-5.

15. Gu J, Kagawa S, Takakura M, Kyo S, Inoue M, Roth JA, et al. Tumor-specific transgene expression from the human telomerase reverse transcriptase promoter enables targeting of the therapeutic effects of the Bax gene to cancers. Cancer Res. 2000;60(19):5359-64.

16. Kitazono M, Goldsmith ME, Aikou T, Bates S, Fojo T. Enhanced adenovirus transgene expression on malignant cells treated with histone deacetylase inhibitor FR901228. Cancer Res. 2001;61(17):6328-30.

17. Siervo-Sassi RR, Marrangoni AM, Feng X, Naoumova N, Sinans M, Edwards RP, et al. Physiological and molecular effects of Apo2L/ TRAIL and cisplatin in ovarian carcinoma cell lines. Cancer Lett. 2003;190(1):61-72.

\section{Discussion}

Dr Jack Roth (Houston, Tex). One interesting aspect, as you pointed out, is TRAIL resistance. I wondered whether you looked at these cell lines with TRAIL protein to see whether you get the same effect in combination with cisplatin, or does the use of this adenovirus construct allow you to overcome TRAIL resistance in resistant cell lines?

Dr Reddy. We did look at the soluble and recombinant forms of TRAIL in combination with cisplatin and saw that we were able to sensitize resistant cell lines to the recombinant soluble TRAIL form using cisplatin pretreatment. That combination was also found to be safe in normal cells at doses of up to $100 \mathrm{ng} / \mathrm{mL}$ soluble TRAIL. We did not observe the ability of the adenovirus construct to overcome recombinant TRAIL resistance.

Dr Roth. Was the magnitude of the effect the same that you saw with the adenovirus TRAIL? 
Dr Reddy. The previous work only looked at the esophageal cancer cell lines, the TE2s, TE12s, and TE3s, only 2 of which I have replicated here. I believe that we saw similar effects, but I cannot attest to the mesotheliomas or the NSCLC lines.

Dr Roth. One final question. Is this a completely scheduledependent effect? In other words, if you treat simultaneously with the cisplatin and your adenovirus TRAIL construct, do you see this synergistic interaction, or if you treat with the cisplatin afterward?

Dr Reddy. We are currently working on the specific pharmacokinetics of what would be the ideal treatment schedule. With concurrent treatment, we do not see this enhancement. We are trying to evaluate whether we need the full 24 hours of pretreatment or whether we can pretreat for only 8 hours. Our goal is to eventually mimic what would be done in a clinical setting.

Dr Roth. And could you just speculate on the mechanism here? Do you think this is an enhancement of adenoviral transduction, or is there some other pathway that is activated by the cisplatin that causes the enhanced TRAIL responsiveness?

Dr Reddy. Specifically for cisplatin, our hypothesis is that the enhanced transduction might be due to the DNA-alkylating effect of the cisplatin itself, causing an increase in the DNA repair machinery of the cell, thus increasing the adenovirus gene expression. Other groups have looked at using histone deacetylase inhibitors as a method of enhancing adenoviral gene expression and have shown an increase in CAR, the Coxsackie virus and adenovirus receptor, expression. For cisplatin though, we think it is related to the DNA repair machinery, but we have not evaluated the CAR expression yet.

Dr Nguyen. I am really honored to have Dr Roth open the discussion of this article because the idea of using cisplatin in combination with adenovirus expression was actually coming from Dr Roth's laboratory when I was his fellow.

Dr Roth, that is a very important question, the third one, concerning the potential mechanisms of this combination. We thought hard about it, and we tried to find a way to dissect out the molecular mechanism. We believe that the increased expression of TRAIL is one mechanism. The other mechanism that we think is happening is that somehow cisplatin enhances the responsiveness of the target cells. Therefore we have 2 populations. One population is cells that infect the virus. The other population is cells that are actually going to be affected by the TRAIL itself. Therefore I think that the cisplatin would do both, and we tried to find a way to dissect it out. Dr Roth touched on a very important fundamental question to this combination.

Dr David R. Jones (Charlottesville, Va). Just to follow up on that, have you looked at any other sensitizing chemotherapy agents at all? And how long does this effect last? The assays you showed were a more short-term kind of apoptotic assay. Do you really know how many cells die after 7 days of treatment?

Dr Reddy. We have looked at other chemosensitizers. We have looked at trichostatin $\mathrm{A}$ in combination with the adenovirus TRAIL, and we are still evaluating that. We have also looked at trichostatin A, which is a histone deacetylase inhibitor, in combination with a recombinant protein soluble TRAIL and seen a significant enhancement there. Therefore I think there are other noncisplatin chemoenhancers that we can use.

In terms of the length of our treatment, most of these were, I think, a total of about 4 to 5 days, even in our apoptosis assays. We have not looked at anything as far out as 7 days, but clearly we have seen a synergistic effect with the combination, even in that short period of time. 